\title{
Third Obtuse Marginal Branch Artery
}

National Cancer Institute

\section{Source}

National Cancer Institute. Third Obtuse Marginal Branch Artery. NCI Thesaurus. Code C102351.

The third artery arising from the left circumflex artery that supplies the lateral wall, when counted from proximal to distal. 